\title{
Optimal Noninvasive Colon Cancer Screening Modality in Patients Not Receiving Colonoscopy
}

\author{
Akshay N. Narendra \\ Saint James School of Medicine, Park Ridge, IL, USA \\ Email: akshay.narendra93@gmail.com
}

How to cite this paper: Narendra, A.N. (2022) Optimal Noninvasive Colon Cancer Screening Modality in Patients Not Receiving Colonoscopy. Advances in Aging Research, 11, 1-15.

https://doi.org/10.4236/aar.2022.111001

Received: November 13, 2021

Accepted: January 24, 2022

Published: January 27, 2022

Copyright $\odot 2022$ by author(s) and Scientific Research Publishing Inc. This work is licensed under the Creative Commons Attribution International License (CC BY 4.0).

http://creativecommons.org/licenses/by/4.0/

\begin{abstract}
Colon cancer is the third most common among cancer deaths in the US for both men and women. The incidence of colonoscopy has been soaring in younger patients, which led to changes in recent United States Preventive Services Task Force (USPSTF) guidelines to reduce the age for screening from 50 years to 45 years. Demand for colonoscopy services is surging due to increased incidences of colorectal cancer (CRC) in the both aging and younger population. Increased referrals have led to an insufficient workforce in hospitals and long waiting lists. Further, results from colonoscopy reveal a low percentage of CRC or another severe bowel disease (SBD). Therefore, colon cancer screening is a growing concern, particularly in patients who otherwise have a very long-life expectancy, and who are most likely to benefit from screening. Another reason to boost CRC screening is to minimize the load on hospitals by reducing the patients that undergo colonoscopy unnecessarily because only a low percentage of CRC occurrence is observed in individuals undergoing colonoscopy. In recent years, there are a variety of screening options available for CRC. Noninvasive alternatives include fecal immunochemical test (FIT), multitarget stool DNA testing (MT-sDNA, available under the brand name Cologuard), computed tomography (CT) colonography (previously called virtual colonoscopy), guaiac-based fecal occult blood testing (gFOBT), and capsule colonoscopy (CC). These tests have varied the degree of evidence supporting their use. This study focuses on the most recent survey and efficacy of noninvasive methods to prevent and detect colorectal cancer (CRC).
\end{abstract}

\section{Keywords}

Colonoscopy, Sigmoidoscopy, Fecal Immunochemical Test, Fecal Occult Blood Test, CT Colonography 


\section{Introduction}

The mortality from cancer has steadily decreased by about $30 \%$ from its peak spanning the period from 1991 through 2018. This gain in lifespan is due to increased cessation in smoking and advances in early detection and therapy [1]. It is about three million fewer deaths compared to the peak year. Such valuable population-based surveillance data is made possible by the National Cancer Institute's (NCI's) Surveillance, Epidemiology, and End Results (SEER) program, and by the Centers for Disease Control and Prevention's (CDC's) National Program of Cancer Registries (NPCR) [1]. The data indicate that CRC incidences declined rapidly during the 2000s owing to wide-spread colonoscopy uptake. Based on the statistical analyses it is estimated that about 50,000 colon and rectal cancer deaths would occur this year in the US. There are limitations on predicted numbers for mortality resulting from modelling studies, however, reliable metrics for tracking cancer incidences and mortality are obtained from the databases such as SEER and National Center for Health Statistics (NCHS).

In general risks for developing CRC depends on a combination of environmental factors, genetic factors, diets, lifestyle, and morbidity. CRC is curative when diagnosed at an early stage and more importantly, it is preventable cancer. Palliative treatment options such as endoscopy, oncologic, and surgical treatments are available to treat advanced stages in cancer [2]. American Cancer Society (ACS) recommends screening colonoscopy for all individuals at the age of 45 years. Table 1 lists the screening recommendations by multi-society task force (MSTF) in 2017 and updated recently [3] [4]. Regarding CRC screening for average-risk individuals, MSTF weakly recommends for individuals ages 45 to 49 years, strongly recommends for individuals 50 years, and above, and weakly recommends stopping at age 75 years [4]. iFOBT, gFOBT, and FIT-DNA are essentially in-house tests

Table 1. CRC screening recommendations by MSTF in 2017 and recent update*.

\begin{tabular}{ll}
\hline Moderate-risk individuals & History of CRC \\
\hline $\begin{array}{l}\text { First-tier option: High-quality }{ }^{\text {a }} \text { colonoscopy at } \\
\text { 10-year intervals or FIT every year. }\end{array}$ & $\begin{array}{l}\text { For persons with two close relative } \\
\text { diagnosed with CRC and with } \\
\text { advanced adenomas. }\end{array}$ \\
$\begin{array}{l}\text { Second-tier option: CT colonography at 5-year } \\
\text { intervals or FIT-fecal DNA test at 3-year intervals } \\
\text { or flexible sigmoidoscopy at 5- or 10-year intervals. }\end{array}$ & $\begin{array}{l}\text { For individuals with one close } \\
\text { relative diagnosed with CRC and }\end{array}$ \\
$\begin{array}{ll}\text { Third-tier option: Capsule colonography at 5-year } \\
\text { intervals. }\end{array}$ & $\begin{array}{l}\text { For individuals with symptoms } \\
\text { and/or age 50 years. }\end{array}$
\end{tabular}

CRC: Colorectal cancer; MSTF: Multi-society task force; FIT: Fecal immunochemical test;

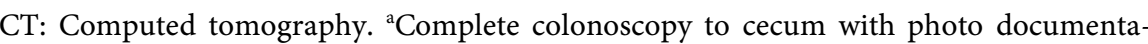
tion. ${ }^{\star}$ From multi-society task force publications [3] [4]. Multi-society task force is comprised of American college of gastroenterology, the American gastroenterological association, and the American society for gastrointestinal endoscopy. The update focuses on the starting age (45 years) and stopping age (75 years) for CRC screening in average-risk individuals. 
because the stool specimens are collected using a kit and sent to laboratories for analysis. If any of these tests or sigmoidoscopy or CT colonography is positive, colonoscopy is indicated. The left-sided (distal) colon has a better detection rate compared to the right-sided (proximal) colon. Risks of developing cancer are more associated with the right-sided colon due to poor detection of polyps in this area. Methods are being developed to increase the sensitivity in the detection [2].

While colonoscopy is the paradigm screening test with dual benefit of being both diagnostic and therapeutic, not all patients are candidates for colonoscopy, due to varied reasons, including anatomic reasons, inability to tolerate sedation, and intolerance of colonoscopy without sedation. Moreover, many patients do not wish to undergo screening, so the question remains as to what the optimal screening choice is for these patients. Colonoscopy is effective for both detecting and removing pre-cancerous lesions. However, individuals have inhibitions to this procedure perhaps due to colon cleansing protocol, pain and complications associated with the procedure, and cost. Colon cleansing regimen has two types-low-volume split-dose and high-volume split-dose regimens. Thorough cleaning is necessary for accurate detection of colon polyps. The cleanliness is estimated by the Boston bowel preparation scale (BBPS). If the colon is not clean repetition of colonoscopy is necessary at regular intervals. High-risk individuals with family histories need surveillance periodically [2].

CRC has high mortality when detected at an advanced stage, however, it is largely preventable with proper and routine care. Therefore, screening is of paramount importance in public health management to decrease CRC risk [5]. CRC is the most treatable cancer if diagnosed in the early stages. Stages I and II CRC is treated by surgical removal of the tumor. Stage III CRC treatment involves surgical resection of the tumor combined with adjuvant chemotherapy. Stage IV CRC treatment includes chemotherapy, biologically targeted therapy, radiotherapy, and others. Several clinical studies are underway for early detection, prevention, and therapy of $\mathrm{CRC}$ at the NCI. At the molecular level mutations on multiple genes are implicated in the development of CRC. It is necessary that CRC should be managed by a team comprised of primary care physicians, gastroenterologists, oncologists, radiologists, surgeons, paramedical team, and educators [2].

There is a need to fulfill the educational needs of primary care doctors, oncologists, pharmacists, and other healthcare professionals who manage patients fighting cancer [6]. CRC is the fourth most diagnosed cancer in the United States. Patients with localized CRC have a $90 \%$ relative 5-year survival rate, whereas those with regional and distant disease have a rate of $71 \%$ and $14 \%$, respectively. $\mathrm{CRC}$ is potentially preventable by detecting its manifestations early on via screening. Regular physical activity, intake of Aspirin, and proper diet could contribute toward decreased colon and/or rectal cancer risk. Smoking and alcohol consumption increases the risk for CRC [6].

Most CRCs are known to develop through the adenoma-carcinoma sequence, so removing it precursor lesions offers an opportunity to prevent cancer. Screen- 
ing efforts use this strategy to remove and detect early-stage CRC. Table 2 shows the various screening strategies and their efficacies [7]. Screening methods are not free from errors, deficiencies, and harms-false positive and false negative results, and complications from colonoscopy-thus proper judgement, skilled and astute doctors, and multiple tests including colonoscopy may be needed at regular intervals. There are established methods in screening modalities and major developments in new strategies would provide a boost in preventing CRC [5]. Data from epidemiology studies show an increase in CRC incidences in patients younger than 50 years is alarming [8] [9] [10] [11].

The highest number of CRC incidences are documented in developed countries while the lowest rates are found in Africa and south-central Asia. This demographic difference may point toward the diets, environmental, and lifestyle aspects [12]. There exist multiple levels of prevention strategy. Primary prevention through healthy diet and lifestyle, and chemoprevention. Secondary prevention

Table 2. Summary of evaluation of CRC screening tests*.

\begin{tabular}{|c|c|c|c|}
\hline Methods & Performance & Advantages & Drawbacks \\
\hline \multicolumn{4}{|l|}{ Stool/blood-based tests } \\
\hline FIT $^{\mathrm{a}}$ (stool) & $\begin{array}{l}79 \% \text { sensitivity } \\
94 \% \text { specificity }\end{array}$ & $\begin{array}{l}\text { Noninvasive; Nocomplications; } \\
\text { Home-based test. }\end{array}$ & $\begin{array}{l}\text { Positive results require colonoscopy; } \\
\text { test repeated annually; low sensitivity } \\
\text { for advanced adenomas; insensitive } \\
\text { to serrated lesions. }\end{array}$ \\
\hline MT-sDNA (stool) & $\begin{array}{l}92 \% \text { sensitivity } \\
87 \% \text { specificity }\end{array}$ & $\begin{array}{l}\text { Noninvasive with no complications; } \\
\text { Home-based test; better sensitivity for } \\
\text { late-stage adenomas and lesions than } \\
\text { FIT alone. }\end{array}$ & $\begin{array}{l}\text { Positive results need colonoscopy; } \\
\text { repeat every } 3 \text { years; more expensive } \\
\text { than FIT alone. Concerns for false } \\
\text { positive. }\end{array}$ \\
\hline Septin-9 (blood) & $\begin{array}{l}48 \% \text { sensitivity } \\
91 \% \text { specificity }\end{array}$ & $\begin{array}{l}\text { Minimally invasive; no complications; } \\
\text { test can be added to usual blood draw. }\end{array}$ & $\begin{array}{l}\text { Low sensitivity for CRC. Requires } \\
\text { colonoscopy for positive results. }\end{array}$ \\
\hline \multicolumn{4}{|l|}{ Visual tests } \\
\hline Colonoscopy & $100 \%$ detection & $\begin{array}{l}\text { Both diagnostic and therapeutic; can } \\
\text { detect cancers and precursor polyps. }\end{array}$ & $\begin{array}{l}\text { Depends on the skill of operator; bowel } \\
\text { preparation and sedation necessary; } \\
\text { Risk of complications }<0.1 \% \text {. }\end{array}$ \\
\hline Flexible sigmoidoscopy & $\begin{array}{l}90 \%-100 \% \text { sensitivity } \\
\text { for distal CRC }\end{array}$ & $\begin{array}{l}\text { Less invasive than colonoscopy; low } \\
\text { risk of complications. }\end{array}$ & $\begin{array}{l}\text { Colonoscopy needed for positive } \\
\text { results; Need to repeat every } 5 \text { - } 10 \\
\text { years; enema preparation is a } \\
\text { pre-requisite. }\end{array}$ \\
\hline CT colonography & $\begin{array}{l}90 \%-100 \% \text { for CRC; } \\
\text { variable sensitivity for } \\
\text { polyps and poor } \\
\text { sensitivity for lesions. }\end{array}$ & $\begin{array}{l}\text { Less invasive than colonoscopy; } \\
\text { sedation not required; lower risk of } \\
\text { complications than colonoscopy. }\end{array}$ & $\begin{array}{l}\text { Colonoscopy for positive results; } \\
\text { bowel preparation is necessary; } \\
\text { trained radiologists are inadequate } \\
\text { across USA. }\end{array}$ \\
\hline Colon capsule & $\begin{array}{l}81 \% \text { sensitivity } \\
93 \% \text { specificity } \\
\text { for polyps } \geq 6 \mathrm{~mm}\end{array}$ & $\begin{array}{l}\text { Minimally invasive. Does not require } \\
\text { sedation; newer generation tests are } \\
\text { home-based. }\end{array}$ & $\begin{array}{l}\text { Colonoscopy needed for positive } \\
\text { results; Requires bowel preparation. }\end{array}$ \\
\hline
\end{tabular}

CRC: Colorectal cancer; FIT: Fecal immunochemical test; ${ }^{a}$ At FIT cutoff of $20 \mathrm{mg} / \mathrm{g}$ of stool. ${ }^{\star S o u r c e}$ is Shaukat et al. [7]. 
through CRC screening via detecting and removing precursor lesions. Tertiary prevention by CRC surveillance by screening at regular intervals [12].

All screening modalities are cheaper compared to dearer costs incurred because of no screening. The costs for CRC treatment for later stage CRC and management are huge with minimal prospects for survival [5]. Prevention is better than cure is a famous adage that gains significance in many ways. Thus, there is an invaluable advantage in screening owing to potential saving of lives, avoiding huge medical bills, and stress on hospital infrastructure.

In the United States, lung cancer registers the highest cancer mortality. CRC is the second leading cause of cancer deaths and is the third most occurring cancer. The reduction in incidences began in 1980s and has declined steeply beginning $21^{\text {st }}$ century. This trend is attributed to early CRC screening, resection of adenomatous or precancerous polyps with colonoscopy, advances in surgical and treatment approaches [2] [7]. FIT-DNA test combines FIT with DNA markers enabling better detection for both advanced adenomas and CRC as opposed to FIT test only [13]. This provides an example of improvement of the existing methods.

Primary care physicians are pivotal in both prevention and early detection of CRC. When patients present with symptoms, they need to appropriately direct the patients for secondary care [14]. Colonoscopy is a gold standard investigation for diagnosing CRC with histological proof of diagnosis through biopsy. According to American college of gastroenterology (AUG) guidelines, screening for average-risk patients is recommended at 50 years by NCCN. Lowering the age could overwhelm the infrastructure and increased liability on insurance companies [6] [7].

\subsection{Search Strategy and Selection Criteria}

Original and most recent review articles related to noninvasive CRC screening were obtained using PubMed and other national library resources such as NCBI. PubMed is a no-cost program that retrieves references and abstracts on life sciences and biomedical subjects. The phrases used in this search were CRC screening, non-invasive methods for CRC screening, preventive methods used for CRC screening, and others. Review articles published in 2020 and 2021 were generally used. Older articles were used if that specific topic was not reviewed in recent years.

\subsection{Screening Options}

CRC screening is vital to reduce mortality rate. Colonoscopy has proved to be highly successful in detecting early on and removal of benign and precancerous lesions. This procedure is invasive, costly, and requires elaborate resource [15]. Hence, there is an urgent need to have CRC screening modalities that are noninvasive, effective, and economical that can be used in conjunction with colonoscopy as the second level of protection. Such complementary strategies may 
cater to the diverse population around the world. Noninvasive screening procedures for CRC are a viable alternative to colonoscopy in certain patient populations [15]. Noninvasive CRC screening modalities include stool-based FIT and MT-sDNA tests, blood-based Septin-9 test, and image-based colon capsule and computed tomography (CT) colonography tests [15]. These strategies are largely underutilized in CRC screening. It is important to recognize that the described noninvasive CRC screening strategies are not a replacement, but a potent complementary and synergistic strategy to conventional colonoscopy. Screening and surveillance remain to be an integral part of CRC patient care [15]. Demand for colonoscopy services is high due to aging and some younger population, leading to inadequate medical personnel to meet the needs of long waiting lists. Nevertheless, results from colonoscopy reveal a low percentage of CRC or other SBD [16]. This finding further reinforces that screening is very important to avoid expensive invasive colonoscopy.

\subsection{Outreach}

Strategies to popularize and enlist patients with risk or symptoms for CRC screening are important. Communication with the Medicaid population via mailed reminders about colorectal cancer (CRC) screening by fecal immunochemical testing (FIT) has been shown to be impressive [17]. In this study, FIT values > $100 \mathrm{ng}$ of hemoglobin per milliliter of buffer were considered positive [18]. Rural populations have more CRC incidences compared to urbanites in USA [19]. There exists a correlation among rurality, screening rates, and mortality. Educating rural residents about the availability of kits, mailing screening kits and instruction to individuals may increase screening rates [20]. A study involving a large cohort of 35,000 unscreened Medicaid enrollees ages 52 to 64 years showed that health departments and Medicaid programs could benefit from low costs by mailing FIT kits to Medicaid population. Such increased CRC screening can prevent mortality and huge medical bills [17]. Public initiatives to boost CRC screening could aid in saving lives from CRC [7].

\subsection{Ongoing Developments}

Genetic, immune, and oncologic researches are underway for early detection and improved management of CRC [2]. Emerging screening tests involving detection of circulating tumor DNA (ctDNA) are promising and can indicate the overall tumor burden [5] [21]. Immune-based therapies such as checkpoint inhibitors are considered to play a vital role in cancer control. An immune-based assay named the "immunoscore" was defined and included as part of the guidelines for the classification of cancer [22]. Developments in artificial intelligence technologies are used to detect polyps [23]. Screening for CRC remains low despite benefits of detecting curable-stage cancerous cells. The U.S. preventive services task force provides no preferential screening test, but options are available to improve uptake of screening. 


\section{Discussion}

\subsection{Screening Methods}

This review aims to analyze and compare the various methods/modalities used in CRC screening. The comparative study seeks to identify strengths and weaknesses of the methods. The literature available in the public domain was used to assess the factors influencing the reduction of the mortality rate. Table 2 broadly classifies the available screening methods based on the procedures used to evaluate CRC. Salient features of each of the methods are described below. Figure 1 shows a schematic diagram of the colon.

\subsection{Stool-Based Tests}

\section{Guaiac-based Fecal Occult Blood Test ( $g F O B T$ )}

This is a stool-based test and uses the pseudoperoxidase activity of heme to detect the presence of blood in the feces. It is inexpensive, easy to perform, and is popular for several decades. However, this suffered from false-positive and false-negative results due to patients' diets. This method is replaced by FIT (see below) because an antibody is used to detect the presence of hemoglobin and is independent of the diet [24] [25].

Fecal Immunochemical Test (FIT)

Quantitative Fecal Immunochemical Tests measure fecal hemoglobin (f-Hb) to detect risk of bowel disease. Patients with $\mathrm{f}-\mathrm{Hb} \geq 400 \mu \mathrm{g} \mathrm{Hb} / \mathrm{g}$ feces are predicted to have $>50 \%$ risk of the significant bowel disease [26]. FIT kits with instructions for patients were used to collect FIT specimen by the general practitioner. $\mathrm{Hb}$ content was measured by an instrument that can detect as low as 2 $\mu \mathrm{g} / \mathrm{g}$ to $400 \mu \mathrm{g} / \mathrm{g}$ of feces. If a patient's $\mathrm{f}-\mathrm{Hb}<10 \mu \mathrm{g} / \mathrm{g}$ of feces and has no iron

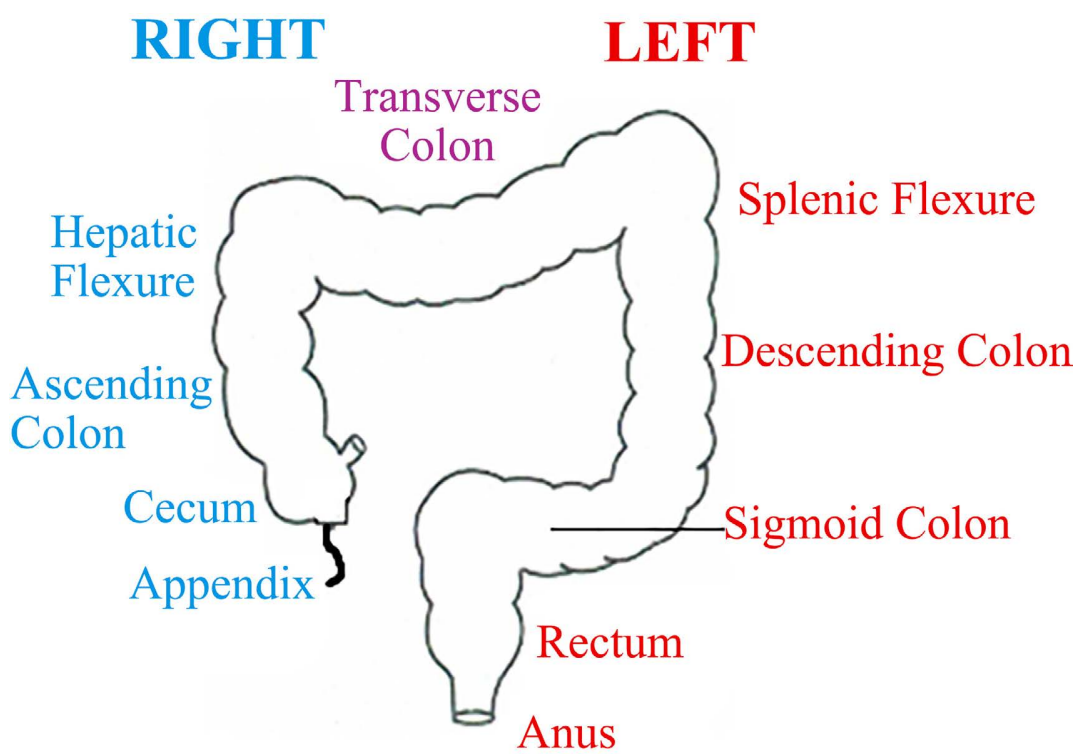

Figure 1. Anatomy of the colon. Different segments and the right and left portions are labelled. The CRC in the right-sided colon is known to be severe and difficult to detect lesions compared to CRC in the left-sided portion. 
deficiency, anemia, or a rectal mass, then CRC is very unlikely. Such tests are routinely available at the primary care level in Scotland for all ages. Such a system ensures diagnosing bowel disease at a very early stage.

An interesting study was conducted to explore if cancer screening procedures (FIT-positive patients receiving diagnostic colonoscopy) result in serious harms. It is important to minimize these harms. A study by Dutch group on this theme concluded that benefits outweigh the diagnostic colonoscopy-related mortality in a cohort of FIT-positive patients [16].

Measurement of fecal hemoglobin with FIT in patients with rectal bleeding can suggest who requires urgent referral for colonoscopy. Patients presenting to primary care about rectal bleeding is an alarm symptom for CRC although it could be for other reasons. To be safe it needs to be considered as a possibility for $\mathrm{CRC}$ and should be either detected or ruled out. $\mathrm{f}-\mathrm{Hb} \geq 10 \mathrm{mg} \mathrm{Hb} / \mathrm{g}$ of feces were characterized as positive and recommended for colonoscopy. If $\mathrm{f}-\mathrm{Hb}<10$ $\mathrm{mg} \mathrm{Hb} / \mathrm{g}$ of feces sigmoidoscopy alone was suggested for further investigation. FIT can be used as a rational and practical approach to analyze the bleeding symptoms. Such information is valuable because there is no need for endoscopy or full colonoscopy and eases the demand and strain on the hospital units [27].

CRC screening programs are cost-effective compared to those of breast and cervix cancers. Two-step screening strategy is used, first step is noninvasive screening, if positive, the second step is investigation through colonoscopy. FIT is a step 1 screening method used commonly. It suffers from false-positive results to a certain degree, so in search of tools with higher specificity, recently estimation of fecal gut microbiota is suggested to be included with FIT for CRC screening. This added test complements FIT and increases the specificity [12].

Multitarget stool DNA Test (MT-sDNA)

CRC cells spill into the stool and their DNA modifications can be detected. This method is noninvasive, involves molecular-level probes, and displays higher sensitivity for the detection of cancer and advanced precancerous lesions. DNA test includes tests for KRAS mutations, aberrant NDRG4 and BMP3 methylations, and $b$-actin a reference gene for human DNA quantity. Immunochemical assay for human hemoglobin was also performed to compare the MT-sDNA test with FIT. Combined results with a value of 183 or more were considered as positive. Studies showed that sensitivity for detecting CRC was $92.3 \%$ with DNA testing compared to $73.8 \%$ with FIT. FIT values of more than $100 \mathrm{ng}$ of $\mathrm{Hb}$ per $\mathrm{ml}$ of buffer were characterized as positive. Although this method detected significantly more cancer cases than did FIT but suffered from larger false positive results. The advantage in this combined modality is that both altered human DNA test and fecal $\mathrm{Hb}$ test were conducted with one sample. Stool was collected prior to colonoscopy screening with no dietary restrictions. All stool specimens were distributed to different laboratories in a blinded fashion. Table 3 shows the efficacy of DNA test over FIT. The data in this table shows that colonoscopy remains to be the ultimate method to detect the lesions at different stages [18]. 
Table 3. Comparative data for MT-stool DNA (MT-sDNA) test, fecal immunochemical test (FIT) and colonoscopy exam

\begin{tabular}{|c|c|c|c|c|c|}
\hline \multirow[t]{3}{*}{ Advanced stage of CRC } & \multirow[t]{3}{*}{$\begin{array}{l}\text { Colonoscopy } \\
(\mathrm{N}=9989)\end{array}$} & \multicolumn{2}{|c|}{$\begin{array}{c}\text { MT-sDNA } \\
(\mathrm{N}=9989)\end{array}$} & \multicolumn{2}{|c|}{$\begin{array}{c}\text { FIT } \\
(\mathrm{N}=9989)\end{array}$} \\
\hline & & positiv & nsitiv & positive & sensitivity \\
\hline & & no. & $\%$ & no. & $\%$ \\
\hline \multicolumn{6}{|l|}{ Colorectal cancer } \\
\hline Any & 65 & 60 & 92 & 48 & 74 \\
\hline Stage I to III & 60 & 56 & 93 & 44 & 73 \\
\hline CRC/high-grade dysplasia & 104 & 87 & 84 & 66 & 64 \\
\hline Advanced precancerous lesions & 757 & 321 & 42 & 180 & 24 \\
\hline \multirow[t]{2}{*}{ Nonadvanced adenoma } & 2893 & 498 & 17 & 220 & 8 \\
\hline & & \multicolumn{2}{|c|}{ specificity } & \multicolumn{2}{|c|}{ specificity } \\
\hline All nonadvanced adenomas & 9167 & 1231 & 87 & 472 & 95 \\
\hline Negative results on colonoscopy & 4457 & 455 & 90 & 162 & 96 \\
\hline
\end{tabular}

$\mathrm{N}$ represents the total number of evaluated participants. Some definitions: sensitivity: percentage of persons with disease who tested positive; specificity: proportion of persons without disease who tested negative; false negative rate: proportion of persons with disease who tested negative; false positive rate: proportion of persons without disease who tested positive. ${ }^{\star}$ Data from the work of Imperiale et al. [18].

The development of CRC is through a multipronged process that involves genetic and epigenetic alterations of the cellular genome. Among epigenetic modifications, DNA methylation is common and is easily detectable [28]. Cologuard is approved by FDA for multi-target stool DNA test that detects KRAS mutation, NDRG4 and BMP3 methylations, b-actin, and includes hemoglobin immunoassay. Epi procolon is blood-based test that examines SEPT9 methylation. DNA methylation serves as clinically useful biomarkers to detect various stages of the CRC as well as precursors of CRC. This test can stratify CRC by assessing stool-based DNA methylation. This approach is promising and is currently being optimized to validate in large populations [29] [30]. Changes in DNA methylation can affect gene expression. Such altered genes contribute to tumor progression and thus serve as epigenetic markers. An assay to detect changes in methylation at the Septing gene in plasma samples is used in CRC screening. Recently some volatile organic compounds that can be detected in altered stool smell from patients are used as biomarkers for CRC detection [5].

\subsection{Direct Visualization}

\section{Computed Tomography Colonography (CTC)}

Computed tomography also known as virtual colonoscopy is used as a diagnostic tool for the detection of colorectal neoplasia. The three-dimensional radiographic images provide accurate detection of polyps-both benign and malignant. It is shown to be helpful in cohorts of patients with several polyps as 
well as in asymptomatic population. The detection sensitivity compares well with optical colonoscopy in identifying adenomatous polyps ranging between 6 to $10 \mathrm{~mm}$ in diameter [31]. CRC is known to develop from benign adenomatous polyps that transform gradually over several years. Therefore, detection and removal of adenomas reduce both the incidence of cancer and mortality.

A small bendable rectal catheter is slipped into the colon and the image of air-filled colon is acquired while the patient is in supine and prone positions using a four-channel or eight-channel CT scanner. Polyps are measured with electronic calipers in the three-dimensional image. Checks for false positive or false negative results in the CT scan and the optical colonoscopy were assessed by radiologists and gastroenterologists. Polyp-matching algorithm was used to detect the polyps with certainty. Statistical analysis was performed on these results from virtual and optical colonoscopy images. Tests of significance include $\mathrm{McNe}$ mar's test, chi-square tests, or paired t-tests [31]. Advances in CT virtual colonoscopy have the potential to be a powerful screening modality for CRC [32]. A large cohort of about 1200 asymptomatic average-risk adults (mean age 58) was screened by CTC. The conclusion from this study was that CT virtual colonoscopy was very helpful for the detection of CRC in asymptomatic adults [32].

Double contrast barium enema (DCBE) and flexible sigmoidoscopy are used in conjunction as complementary diagnostic tests for colorectal neoplasia. Sigmoidoscopy provides a view of the distal portion (left side of the colon) of the colorectum and access to biopsy and/or remove polyps [5]. CT colonography has replaced DCBE for colorectal evaluation. Studies support that CT colonography is more sensitive than flexible sigmoidoscopy. CTC is a preferred screening method that is economical and minimally invasive with high sensitivity to detect polyps in colon [33].

\section{Colon Capsule endoscopy}

Colon capsule endoscopy involves ingestion of a large pill-sized camera (video capsule), which then transmits images of the whole colon and rectum during its transit. The quality and completeness of colonoscopies could be limited by suboptimal visualization or the scopes' limitation to reach the ascending column, so it is terminated necessitating further evaluation of the colon. CTC is a well-established diagnostic tool often used after such incomplete colonoscopy. The meta-analysis of CRC and CCE indicates CCE is a viable alternative to be introduced for clinical use in conjunction or as an alternative to CTC due to its high diagnostic yield especially in detecting small polyps $<5 \mathrm{~mm}$ [34]. Colon Capsule endoscopy is suggested to be used as a filter test in CRC screening. This may eliminate the need for surveillance colonoscopies in many patients and thus reduce pressure on the hospitals and costs [35]. CTC and CCE are both non-endoscopic methods.

\section{Colonoscopy}

It is a one-step screening. Colonoscopy is the procedure recommended after other non-invasive or less-invasive tests are positive. It is also used for primary screening. It requires bowel preparation and sedation is required in most cases. 
Patients require assistance to transport them after the procedure. Colonoscopy is normally considered as an ultimate procedure to detect CRC although it can miss lesions as well. The procedure confers long-term protection from CRC incidence and mortality [5] [7]. This is the ultimate pathway of all CRC screening tests and most effective single-application method for preventing CRC. Physicians performing colonoscopy must bear the responsibility of endoscopists to perform colonoscopies with skill and adept. Clearly, there exists synergy and complementarity in screening methods.

\section{Current and Future Developments}

CRC is essentially an age-related process. This paper reviews non-invasive methods available to date for colon cancer screening. CRC screening includes both non-invasive and invasive methods. As the incidences occur in all populations relatively young and old, it is necessary to initiate screening at an early age about 45 to 50 years. Since colonoscopy is an invasive method and is performed in the hospital, it requires adequate infrastructure, is expensive, and is not available in many communities around the world. Therefore, prior screening of high-risk or moderate-risk individuals would benefit healthcare units. Non-invasive methods should be popularized in rural areas and outreach efforts should be encouraged to avoid unnecessary mortality, medical expenses, and liability on the government health agencies.

Treatment of advanced stage CRC is expensive and survival rate is slim. In line with the adage "prevention is better than cure" priority should be given to prevention and that is possible through non-invasive, easy-to-use, and inexpensive methods. Although there are disadvantages wherein false negative or false positive results are obtained in the screening tests but can be detected by performing regular and multiple tests that are complementary. These tests could be included along with other routinely conducted hosts of tests such as monitoring cholesterol, glucose, and A1C levels. The key to prevent the dreadful disease is to avail all options both invasive and non-invasive. The optimum strategy is to customize and hybridize the available options to individual patients depending on their likes, dislikes, access to insurance, affordability, availability of medical technology and expertise. This entails primary care physicians and health educators in the community to be proactive to convince the patients to undergo suitable screening tests. This review emphasizes the availability of a spectrum of non-invasive methods. It may be noted that although colonoscopy is robust in its therapeutic value to $\mathrm{CRC}$, it is dependent on the skills of the surgeon and complete colonoscopy, that is, the colonoscope reaching to cecum which is difficult. Therefore, there is a possibility of missing the cancerous polyps so combining with other non-invasive methods would be highly beneficial and complementary. Thus, non-invasive methods have virtues as standalone tools as well as in combination with other related techniques.

Statistical analysis and meta-analysis indicate that non-invasive methods are 
beneficial to patients to prevent CRC. In general, cancer is described as the king of all maladies owing to its indomitable nature and rapid proliferation. So, prevention or at least detection early on is the wisest and best strategy as the treatments are not vigorous to add several life-years to patients. This study emphasizes the role of noninvasive CRC screening has in alleviating suffering and mortality. Population-based surveillance data indicates that breakthroughs and developments in screening and treatment have reduced the mortality rate significantly compared to the past.

Emerging research findings from a wide range of disciplines including basic science, clinical science, biomedical engineering, population surveillance, and others shall improve prevention strategy and curative methods for CRC. A better understanding of molecular and cellular mechanisms involved in carcinogenesis shall help to develop enhanced specific and sensitive methods to detect the precursors of lesions at the onset. Rapid advances in nanomedicine may hold promise to detect and site-specific treatment of CRC seamlessly. Progress in immune-system-based surveillance and therapy would be breakthroughs in the future. Research outcomes on the impact of environmental factors, diet, lifestyles, and genetics to CRC would be invaluable to prevent the disease in the first place. A vigorous three-pronged approach with a focus on prevention, detection, and treatment of CRC will help alleviate or perhaps eliminate the anguish.

\section{Acknowledgements}

Author is thankful to Dr. Seema Elahi for her mentorship and guidance.

\section{Conflicts of Interest}

The author declares no conflicts of interest regarding the publication of this paper.

\section{References}

[1] Siegel, R.L., Miller, K.D., Fuchs, H.E. and Jemal, A. (2021) Cancer Statistics, 2021. CA: A Cancer Journal for Clinicians, 71, 7-33. https://doi.org/10.3322/caac.21654

[2] Ahmed, M. (2020) Colon Cancer: A Clinician's Perspective in 2019. Gastroenterology Research, 13, 1-10. https://doi.org/10.14740/gr1239

[3] Rex, D.K., Boland, C.R., Dominitz, J.A., Giardiello, F.M., Johnson, D.A., Kaltenbach, T., et al. (2017) Colorectal Cancer Screening: Recommendations for Physicians and Patients from the U.S. Multi-Society Task Force on Colorectal Cancer. Gastrointestinal Endoscopy, 86, 18-33. https://doi.org/10.1016/j.gie.2017.04.003

[4] Patel, S.G., May, F.P., Anderson, J.C., Burke, C.A., Dominitz, J.A., Gross, S.A., et al. (2022) Updates on Age to Start and Stop Colorectal Cancer Screening: Recommendations from the U.S. Multi-Society Task Force on Colorectal Cancer. Gastroenterology, 162, 285-299. https://doi.org/10.1053/j.gastro.2021.10.007

[5] Ladabaum, U., Dominitz, J.A., Kahi, C. and Schoen, R.E. (2020) Strategies for Colorectal Cancer Screening. Gastroenterology, 158, 418-432.

[6] Provenzale, D., Ness, R.M., Llor, X., Weiss, J.M., Abbadessa, B., Cooper, G, et al. 
(2020) NCCN Guidelines Insights: Colorectal Cancer Screening, Version 2.2020. Journal of the National Comprehensive Cancer Network, 18, 1312-1320. https://doi.org/10.6004/jnccn.2020.0048

[7] Shaukat, A., Kahi, C.J., Burke, C.A., Rabeneck, L., Sauer, B.G. and Rex, D.K. (2021) ACG Clinical Guidelines: Colorectal Cancer Screening 2021. The American Journal of Gastroenterology, 116, 458-479. https://doi.org/10.14309/ajg.0000000000001122

[8] Stoffel, E.M. and Murphy, C.C. (2020) Epidemiology and Mechanisms of the Increasing Incidence of Colon and Rectal Cancers in Young Adults. Gastroenterology, 158, 341-353.

[9] Burnett-Hartman, A.N., Lee, J.K., Demb, J. and Gupta, S. (2021) An Update on the Epidemiology, Molecular Characterization, Diagnosis, and Screening Strategies for Early-Onset Colorectal Cancer. Gastroenterology, 160, 1041-1049.

[10] Altieri, M.S., Thompson, H., Pryor, A., Yang, J., Zhu, C., Talamini, M., et al. (2021) Incidence of Colon Resections Is Increasing in the Younger Populations: Should an Early Initiation of Colon Cancer Screening be Implemented? Surgical Endoscopy, 35, 3636-3641. https://doi.org/10.1007/s00464-020-07842-8

[11] Ohri, A., Robinson, A., Liu, B., Bhuket, T. and Wong, R. (2020) Updated Assessment of Colorectal Cancer Incidence in the U.S. by Age, Sex, and Race/Ethnicity. Digestive Diseases and Sciences, 65, 1838-1849.

https://doi.org/10.1007/s10620-019-05913-y

[12] Malagón, M., Ramió-Pujol, S., Serrano, M., Amoedo, J., Oliver, L., Bahí, A., et al. (2020) New Fecal Bacterial Signature for Colorectal Cancer Screening Reduces the Fecal Immunochemical Test False-Positive Rate in a Screening Population. PLoS ONE, 15, e0243158. https://doi.org/10.1371/journal.pone.0243158

[13] Hoffman, R.M., Levy, B.T. and Allison, J.E. (2021) Rising Use of Multitarget Stool DNA Testing for Colorectal Cancer. JAMA Network Open, 4, e2122328. https://doi.org/10.1001/jamanetworkopen.2021.22328

[14] Calanzani, N., Chang, A., Van Melle, M., Pannebakker, M.M., Funston, G. and Walter, F.M. (2021) Recognising Colorectal Cancer in Primary Care. Advances in Therapy, 38, 2732-2746. https://doi.org/10.1007/s12325-021-01726-6

[15] Lou, S. and Shaukat, A. (2021) Noninvasive Strategies for Colorectal Cancer Screening: Opportunities and Limitations. Current Opinion in Gastroenterology, 37, 44-51. https://doi.org/10.1097/MOG.0000000000000688

[16] Kooyker, A.I., Toes-Zoutendijk, E., Opstal-Van Winden, A.W.J., Buskermolen, M., van Vuuren, H.J., Kuipers, E.J., et al. (2021) Colonoscopy-Related Mortality in a Fecal Immunochemical Test-Based Colorectal Cancer Screening Program. Clinical Gastroenterology and Hepatology, 19, 1418-1425.

[17] Wheeler, S.B., O’Leary, M.C., Rhode, J., Yang, J.Y., Drechsel, R., Plescia, M., et al. (2020) Comparative Cost-Effectiveness of Mailed Fecal Immunochemical Testing (FIT)-Based Interventions for Increasing Colorectal Cancer Screening in the Medicaid Population. Cancer, 126, 4197-4208. https://doi.org/10.1002/cncr.32992

[18] Imperiale, T.F., Ransohoff, D.F., Itzkowitz, S.H., Levin, T.R., Lavin, P., et al. (2014) Multitarget stool DNA Testing for Colorectal-Cancer Screening. New England Journal of Medicine, 370, 1287-1297. https://doi.org/10.1056/NEJMoa1311194

[19] Carmichael, H., Cowan, M., McIntyre, R. and Velopulos, C. (2020) Disparities in Colorectal Cancer Mortality for Rural Populations in the United States: Does Screening Matter? The American Journal of Surgery, 219, 988-992.

https://doi.org/10.1016/j.amjsurg.2019.09.027 
[20] Sutton, A.L., Preston, M.A., Thomson, M., Litzenberg, C., Taylor, T.F., Cole, E.P., et al. (2021) Reaching Rural Residents to Identify Colorectal Cancer Education and Intervention Targets. Journal of Cancer Education, 36, 338-344. https://doi.org/10.1007/s13187-019-01635-X

[21] Myśliwiec, P., Choromanska, B. and Dadan, J. (2020) Markers of Metastatic Colorectal Cancer. Gastroenterology Review, 15, 94-97.

https://doi.org/10.5114/pg.2019.84848

[22] Bruni, D., Angell, H.K. and Galon, J. (2020) The Immune Contexture and Immunoscore in Cancer Prognosis and Therapeutic Efficacy. Nature Reviews Cancer, 20, 662-680. https://doi.org/10.1038/s41568-020-0285-7

[23] Nazarian, S., Glover, B., Ashrafian, H., Darzi, A. and Teare, J. (2021) Diagnostic Accuracy of Artificial Intelligence and Computer-Aided Diagnosis for the Detection and Characterization of Colorectal Polyps: Systematic Review and Meta-Analysis. Journal of Medical Internet Research, 23, e27370.

[24] Meklin, J., Syrjänen, K. and Eskelinen, M. (2020) Colorectal Cancer Screening with Traditional and New-Generation Fecal Immunochemical Tests: A Critical Review of Fecal Occult Blood Tests. Anticancer Research, 40, 575-581. https://doi.org/10.21873/anticanres.13987

[25] Meklin, J., SyrjÄnen, K. and Eskelinen, M. (2020) Fecal Occult Blood Tests in Colorectal Cancer Screening: Systematic Review and Meta-Analysis of Traditional and New-Generation Fecal Immunochemical Tests. Anticancer Research, 40, 3591-3604.

[26] Strachan, J.A. and Mowat, C. (2021) The Use of Faecal Haemoglobin in Deciding Which Patients Presenting to Primary Care Require Further Investigation (and How Quickly)-The FIT Approach. EJIFCC-Electronic Journal of the IFCC, 32, 52-60.

[27] Digby, J., Strachan, J.A., McCann, R., Steele, R.J., Fraser, C.G. and Mowat, C. (2020) Measurement of Faecal Haemoglobin with a Faecal Immunochemical Test Can Assist in Defining Which Patients Attending Primary Care with Rectal Bleeding Require Urgent Referral. Annals of Clinical Biochemistry, 57, 325-327. https://doi.org/10.1177/0004563220935622

[28] Andersen, G.B. and Tost, J. (2018) A Summary of the Biological Processes, Disease-Associated Changes, and Clinical Applications of DNA Methylation. In: Tost, J., Ed., DNA Methylation Protocols, Humana Press, New York, 3-30. https://doi.org/10.1007/978-1-4939-7481-8_1

[29] Raut, J.R., Guan, Z., Schrotz-King, P. and Brenner, H. (2020) Fecal DNA Methylation Markers for Detecting Stages of Colorectal Cancer and Its Precursors: A Systematic Review. Clinical Epigenetics, 12, Article No. 122. https://doi.org/10.1186/s13148-020-00904-7

[30] Olson, J.E., Kirsch, E.J., Edwards, V.D.K., Kirt, C.R., Kneedler, B., Laffin, J.J., et al. (2020) Colorectal Cancer Outcomes after Screening with the Multi-Target Stool DNA Assay: Protocol for a Large-Scale, Prospective Cohort Study (the Voyage Study). BMJ Open Gastroenterology, 7, e000353.

https://doi.org/10.1136/bmjgast-2019-000353

[31] Pickhardt, P.J., Choi, J.R., Hwang, I., Butler, J.A., Puckett, M.L., Hildebrandt, H.A., et al. (2003) Computed Tomographic Virtual Colonoscopy to Screen for Colorectal Neoplasia in Asymptomatic Adults. New England Journal of Medicine, 349, 2191 2200. https://doi.org/10.1056/NEJMoa031618

[32] Plattner, D. (2004) Computed Tomographic Virtual Colonoscopy to Screen for Colorectal Neoplasia in Asymptomatic Adults. European Surgery, 36, 121-122. https://doi.org/10.1007/s10353-004-0078-3

[33] Pickhardt, P.J. (2012) Diagnostic Yield of Flexible Sigmoidoscopy and CTC. Nature 
Reviews Gastroenterology \& Hepatology, 9, 195-196. https://doi.org/10.1038/nrgastro.2012.40

[34] Deding, U., Kaalby, L., Boggild, H., Plantener, E., Wollesen, M.K., Kobaek-Larsen, M., et al. (2020) Colon Capsule Endoscopy vs. CT Colonography Following Incomplete Colonoscopy: A Systematic Review with Meta-Analysis. Cancers, 12, Article No. 3367. https://doi.org/10.3390/cancers12113367

[35] Kaalby, L., Deding, U., Kobaek-Larsen, M., Havshoi, A.V., Zimmermann-Nielsen, E., Thygesen, M.K., et al. (2020) Colon Capsule Endoscopy in Colorectal Cancer Screening: A Randomised Controlled Trial. BMJ Open Gastroenterology, 7, e000411.

https://doi.org/10.1136/bmjgast-2020-000411

\section{List of Abbreviations}

ACG: American College of Gastroenterology;

ACS: American Cancer Society;

BBPS: Boston Bowel Preparation Scale;

CC: Capsule Colonoscopy;

CCE: Colon Capsule Endoscopy;

CDC: Centers for Disease Control and Prevention;

CRC: Colorectal Cancer;

CT: Computed Tomography;

CTC: CT Colonography;

DCBE: Double Contrast Barium Enema;

FIT: Fecal Immunochemical Test;

gFOBT: guaiac-based Fecal Occult Blood Testing;

MSTF: Multi-Society Task Force;

MT-sDNA: Multitarget stool DNA testing;

NCBI: National Center for Biotechnology Information;

NCCN: National Comprehensive Cancer Network;

NCHS: National Center for Health Statistics;

NCI: National Cancer Institute;

NPCR: National Program of Cancer Registries;

SBD: Severe Bowel Disease;

SEER: Surveillance, Epidemiology, and End Results;

USPSTF: United States Preventive Services Task Force. 